\title{
Effects of miR-21 on proliferation and apoptosis of WT cells via PTEN/Akt pathway
}

\author{
XIULI ZHANG, CHUNYAN LIU, HAIYAN LI and LI GUO \\ Pediatrics Intensive Care Unit, Shanxian Central Hospital, Heze, Shandong 274300, P.R. China
}

Received October 18, 2019; Accepted December 13, 2019

DOI: $10.3892 / \mathrm{etm} .2019 .8376$

\begin{abstract}
Micro ribonucleic acid (miR)-21 in the proliferation and apoptosis of Wilms' tumor (WT) cells was explored. SK-NEP-1 cells were transfected with miR-21 inhibitor to silence the expression of miR-21. Then, the effects of miR-21 silencing on the proliferation and apoptosis of WT SK-NEP-1 cells were detected through cell counting kit-8 (CCK-8), colony formation assay and flow cytometry. The targets of miR-21 were analyzed via TargetScan database. Fluorescence real-time quantitative polymerase chain reaction (RT-qPCR) assay and western blot analysis were conducted to detect the changes in messenger RNA (mRNA) and protein expression levels of gene of phosphate and tension homology deleted on chromosome ten (PTEN) after silencing miR-21. Whether miR-21 directly binds to PTEN was examined by activity detection via dual luciferase reporter gene assay. Western blotting was employed to detect the correlation of miR-21 with PTEN and protein kinase B (Akt). Compared with normal control (NC) group, miR-21 inhibitor group had significantly inhibited proliferation of SK-NEP-1 cells $(\mathrm{P}<0.05)$, notably reduced number of clones $(\mathrm{P}<0.05)$ and overtly raised proportion of apoptotic cells $(\mathrm{P}<0.05)$. The suppression of miR-21 expression upregulated the mRNA and protein expression levels of PTEN, and the results of activity detection via dual luciferase reporter gene assay indicated that miR-21 bound to PTEN 3'-untranslated region (UTR) to repress its expression $(\mathrm{P}<0.05)$. PTEN silencing increased phosphorylated Akt (p-Akt) level in SK-NEP-1 cells, but there was no changes in Akt protein level. After silencing both PTEN and miR-21, the decrease in p-Akt was reversed, thereby reversing the inhibitory effect of miR-21 on the proliferation of SK-NEP-1 cells $(\mathrm{P}<0.05)$. miR-21 affects the proliferation and apoptosis of WT SK-NEP-1 cells via the PTEN/Akt pathway.
\end{abstract}

Correspondence to: $\mathrm{Dr} \mathrm{Li}$ Guo, Pediatrics Intensive Care Unit, Shanxian Central Hospital, No 1 Wenhua Road, Heze, Shandong 274300, P.R. China

E-mail: 13869738669@163.com

Key words: WT, miR-21, PTEN, proliferation, apoptosis

\section{Introduction}

Wilms' tumor (WT), a malignant embryonic tumor derived from nephridioblasts, accounts for $\sim 87 \%$ of childhood kidney tumors, of which the peak age is 3-4 years, and $80 \%$ of patients are aged $<5$ years (1). Such a tumor, first described by Rance in 1814, is named as Wilms' tumor since Max Wilms further described its characteristics in 1899 (2). As to its treatment, surgical resection is mainly adopted at present. Besides, WT is sensitive to radiotherapy, postoperative radiotherapy can improve the efficacy (3), while preoperative chemotherapy is able to decrease the size of the tumor, reduce the risk of surgery, and increase the rate of complete resection. The prognosis of WT depends on histological type, tumor stage, patient age and biological characteristics (4), and is good in most WT patients. After systemic treatment, the 4-year survival rate of patients with WT at any stage is $\sim 54.8-90 \%$ (5). However, there are no special precautions for WT. Therefore, the detection and treatment as early as possible are important for the improvement of the prognosis of WT patients.

Micro ribonucleic acids (miRNAs) pair with their messenger RNAs (mRNAs) to induce post-transcriptional inhibition on their target genes, which are widely involved in modulating most physiological processes such as apoptosis, proliferation, survival, cell cycle and differentiation (6). Considering that $>50 \%$ of miRNAs are located in cancer-related genomic regions or vulnerable sites, they are of importance in the pathogenesis of many types of cancer, including WT. For instance, Ludwig et al (7) identified the miRNA expression profiles in 36 WT tissues and 4 normal kidney tissues via microarray and discovered that miR-183, miR-301a/b and miR-335 are upregulated in embryonic subtypes, and miR-181b, miR-223 and miR-630 are raised in regression subtypes. Jiang et al (8) proved that miR-1180 is overexpressed in WT tissues, and its knockdown induces apoptosis of SK-NEP-1 cells and reduces tumor growth in nude mice. Liu et al (9) collected WT tissues and adjacent normal tissues from WT patients and confirmed that the knockdown of miR-19b evidently represses the proliferation, invasion and migration of WT SK-NEP-1 cells, and the inhibition of miR-19b suppresses the progression of WT by modulating the gene of phosphate and tension homology deleted on chromosome ten (PTEN)/phosphatidylinositol 3-kinase (PI3K)/protein kinase B (Akt) signaling pathway. All of the above findings suggest that miRNAs play important roles in the development and progression of WT. 
It has been confirmed that miR-21 is overexpressed in almost all solid tumors studied, accounting for $15-25 \%$ of total miRNAs in tumor cells and serves as a proto-oncogene (10). Moreover, miR-21 can negatively regulate various target genes, such as programmed cell death 4 (PDCD-4) (11). Given this, miR-21 is a vital participant promoting the proliferation and differentiation of certain tumor cells. However, the studies on the role of miR-21 in WT are rare. Hence, in this study, the effects of miR-21 on the proliferation and apoptosis of WT were investigated.

\section{Materials and methods}

Materials. Roswell Park Memorial Institute (RPMI)-1640 medium (HyClone; GE Healthcare Life Sciences), fetal bovine serum (FBS) (Gibco; Thermo Fisher Scientific, Inc.), M-MLV reverse transcriptase (Promega Corporation), apoptosis assay kit (Sigma-Aldrich; Merck KGaA), 2X Ultra SYBR Mixture kit (Takara Bio), Lipofectamine 2000 (Invitrogen; Thermo Fisher Scientific, Inc.), cell counting kit-8 (CCK-8) reagents (Dojindo), psiCHECK ${ }^{\mathrm{TM}}$ plasmids and dual luciferase kit (Promega Corporation), PTEN, Akt, phosphorylated Akt (p-Akt) antibodies (Cell Signaling Technology, Inc.), CFX96 sequence detection system (Bio-Rad Laboratories, Inc.) and flow cytometer (BD Biosciences).

Cell culture and miRNA transfection. Human WT SK-NEP-1 cell line was from American Type Culture Collection (ATCC). The SK-NEP-1 cells were inoculated in RPMI-1640 medium containing $10 \% \mathrm{FBS}$ and cultured in a $5 \% \mathrm{CO}_{2}$ incubator at $37^{\circ} \mathrm{C}$. miR-21 mimic and inhibitor as well as si-PTEN were designed and synthesized by RiboBio, and then transfected into the SK-NEP-1 cells according to the transfection instructions of Lipofectamine 2000.

Real-time quantitative polymerase chain reaction ( $R T-q P C R)$ assay. Total RNAs were extracted from the SK-NEP-1 cells using TRIzol (Invitrogen; Thermo Fisher Scientific, Inc.), reverse transcribed into complementary deoxyribonucleic acids (cDNAs) using the M-MLV reverse transcriptase (Promega Corporation) as per the instructions of the reverse transcription kit. RT-qPCR was carried out on a CFX96 sequence detection system with $20 \mathrm{ng}$ of cDNA using the 2X Ultra SYBR Mixture kit. The thermal cycle: initial denaturation at $95^{\circ} \mathrm{C}$ for $1 \mathrm{~min}$, denaturation at $94^{\circ} \mathrm{C}$ for $30 \mathrm{sec}$, annealing at $58^{\circ} \mathrm{C}$ for $30 \mathrm{sec}$ and extension at $72^{\circ} \mathrm{C}$ for $10 \mathrm{sec}$ for a total of 30 cycles, and then at $72^{\circ} \mathrm{C}$ for $2 \mathrm{~min}$ and at $16^{\circ} \mathrm{C}$ for $5 \mathrm{~min}$. The primer sequences are: $\mathrm{miR}-21$ : F, $5^{\prime}-\mathrm{GCC}$ AGGCATAGCTTATCAGACTG-3' and R, 5'-CCACTG TCTAGCACGACACTAA-3'; PTEN: F, 5'-AAAGGGACGAA CTGGTGTAATG-3' and R, 5'-TGGTCCTTACTTCCCCATA GAA-3'; U6: F, 5'-GCTTCGGCAGCACATATACTAAAAT-3' and R, 5'-CGCTTCACGAATTTGCGTGTCAT-3'; and GAPDH: F, 5'-CGGAGTCAACGGATTTGGTCGTAT-3' and 5'-AGCCTT CTCCATGGTGGTGAAGAC-3'.

Cell proliferation assay. The proliferation ability of SK-NEP-1 cells transfected with miR-21 inhibitor or control was detected using the CCK-8. The cells were seeded into a 96-well plate at a density of $2 \times 10^{3}$ cells/well and transfected for 24,48 and
$72 \mathrm{~h}$ as described above. Then, the cells were added with $10 \mu \mathrm{l}$ of CCK-8 solution and incubated in an incubator for $3 \mathrm{~h}$. A microplate reader was utilized to read the optical density (OD) value at a wavelength of $450 \mathrm{~nm}$. Colony formation assay was conducted to determine the clonogenic capacity of the SK-NEP-1 cells transfected with miR-21 inhibitor or control. The SK-NEP-1 cells transfected for $24 \mathrm{~h}$ were re-inoculated into a 6-well plate at 500 cells/well for 2 weeks of culture. Paraformaldehyde was added to fix the viable clones, followed by staining with $0.5 \%$ crystal violet at $37^{\circ} \mathrm{C}$ for $1 \mathrm{~h}$. Thereafter, viable cells were counted, and images were captured with a digital camera.

Apoptosis assay. The SK-NEP-1 cells were seeded into a 6-well plate for 1 day and then transfected for $48 \mathrm{~h}$. Next, the cells were trypsinized, harvested, washed twice with cold phosphate buffered saline (PBS), and then re-suspended in $1 \mathrm{X}$ binding buffer. Thereafter, the cell suspension ( $1 \times 10^{5}$ cells) was added with Annexin $\mathrm{V}$ and propidium iodide solution at a ratio of $100 \mu \mathrm{l}: 5 \mu \mathrm{l}$, mixed and let stand in a dark place at room temperature for $15 \mathrm{~min}$. The suspension was added with $1 \mathrm{X}$ binding buffer and loaded on the instrument for detection. Each assay was repeated 3 times independently.

Western blot analysis. The SK-NEP-1 cells were collected and lysed in HEPES lysis buffer containing protease inhibitors. The bicinchoninic acid (BCA) protein concentration assay kit (Pierce; Thermo Fisher Scientific, Inc.) was employed to quantify the total protein concentration. An equal amount of $50 \mu \mathrm{g}$ of total protein was separated by $10 \%$ sodium dodecyl sulphate-polyacrylamide gel electrophoresis (SDS-PAGE) and transferred onto a polyvinylidene fluoride (PVDF) membranes (EMD Millipore). The membrane with separated proteins was blocked with $5 \%$ non-fat milk powder at room temperature for $1 \mathrm{~h}$, incubated with the corresponding primary antibodies at $4{ }^{\circ} \mathrm{C}$ overnight, washed with Tris-buffered saline with Tween-20 (TBST) 3 times, and incubated with the secondary antibodies at $4^{\circ} \mathrm{C}$ for $1 \mathrm{~h}$. Target protein expression bands were visualized through electrochemiluminescence.

Detection of luciferase activity. TargetScan was employed to predict the targets of miR-21. In the luciferase reporter gene assay, the 3'-untranslated region (UTR) was designed based on bioinformatics software. The PTEN 3'-UTR sequences containing predicted miR-21 binding sites and corresponding mutation sites were subjected to PCR amplification and then inserted into the psiCHECK ${ }^{\mathrm{TM}}$ vector together with the downstream luciferase genes. SK-NEP-1 cells were cultured in a 24-well plate at a density of $2.5 \times 10^{5}$ cells/wells, and then co-transfected with PTEN wild-type (WT) or mutant (MUT) reporter plasmids (final concentration: $10 \mathrm{nM}$ ) and miR-21 mimic or control, and $48 \mathrm{~h}$ later, the dual luciferase assay kit was utilized to determine the luciferase activity.

Statistical analysis. Data were expressed as mean \pm standard deviation. Differences between two groups were analyzed by using the Student's t-test. Comparison between multiple groups was done using One-way ANOVA test followed by post hoc test (least significant difference). $\mathrm{P}<0.05$ was considered to indicate a statistically significant difference. 
A

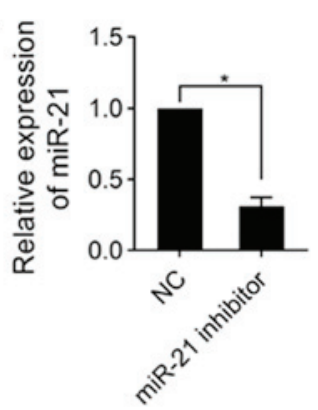

C

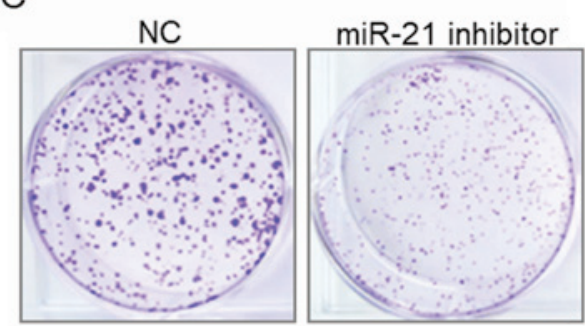

B

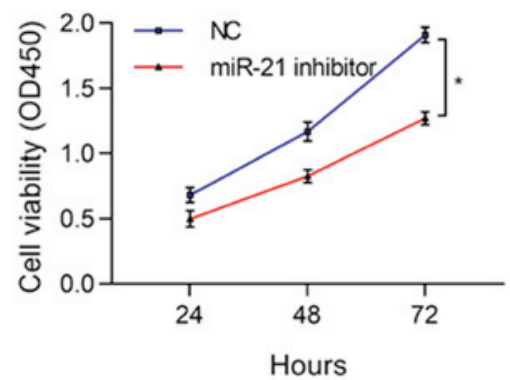

Hours

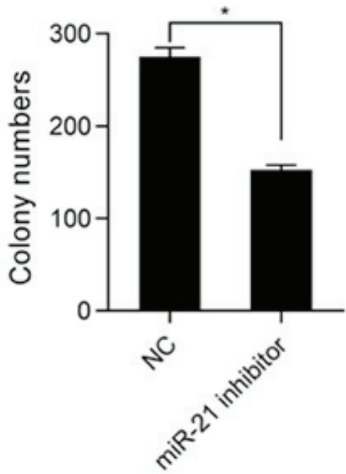

Figure 1. Effects of miR-21 silencing on proliferation ability of WT SK-NEP-1 cells. (A) Silencing effect on miR-21 detected via RT-qPCR. (B) Proliferation ability of SK-NEP-1 cells in miR-21 inhibitor group and NC group determined through CCK-8. (C) Clonogenic capacity of SK-NEP-1 cells in miR-21 inhibitor group and $\mathrm{NC}$ group analyzed by colony formation assay. ${ }^{*} \mathrm{P}<0.05$. miR, micro ribonucleic acid; RT-qPCR, real-time quantitative polymerase chain reaction; WT, Wilms' tumor; NC, normal control; CCK-8, cell counting kit-8.

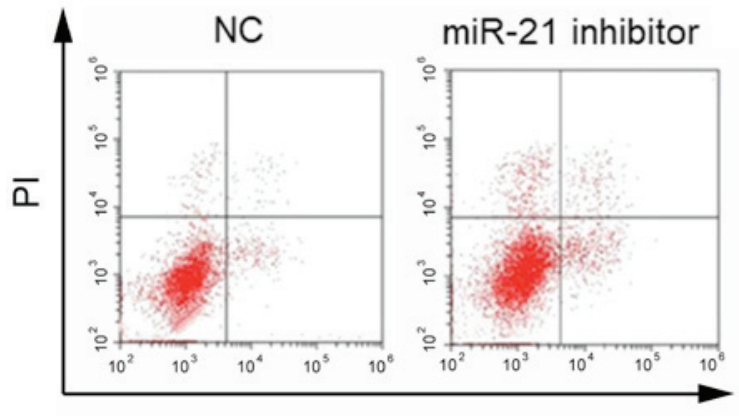

Annexin V - FITC

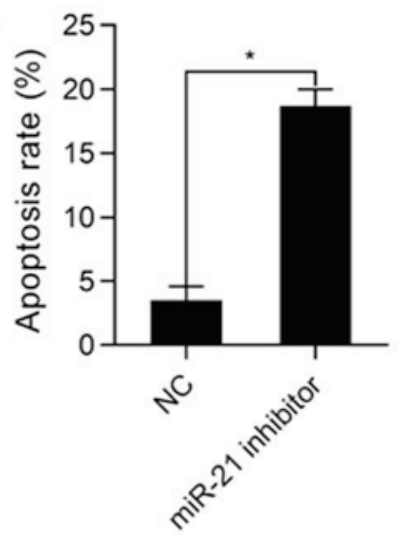

Figure 2. Effects of miR-21 silencing on apoptosis of WT SK-NEP-1 cells determined through flow cytometry. *P<0.05. miR, micro ribonucleic acid; WT, Wilms' tumor.

\section{Results}

Silencing miR-21 inhibits proliferation of SK-NEP-1 cells. To investigate the key role of miR-21 in the proliferation of WT cells, miR-21 inhibitor was transfected into SK-NEP-1 cells to silence the expression of miR-21. The results (Fig. 1A) of RT-qPCR analysis showed that at $48 \mathrm{~h}$ after transfection, the expression of miR-21 was remarkably decreased in SK-NEP-1 cells $(\mathrm{P}<0.05)$, demonstrating that the expression of miR-21 was silenced, so in vitro studies can be conducted. Next, CCK-8 assay was carried out to explore the influence of silencing miR-21 on the proliferation of WT SK-NEP-1 cells, and it was found that the proliferation ability of SK-NEP-1 cells was clearly repressed in miR-21 inhibitor group compared with that in normal control (NC) group $(\mathrm{P}<0.05)$ (Fig. 1B), which was further verified through colony formation assay subsequently. The results (Fig. 1C) revealed that the number of clones was obviously lower in miR-21 inhibitor group than that in NC group $(\mathrm{P}<0.05)$.

Effects of miR-21 silencing on apoptosis of SK-NEP-1 cells. The role of miR-21 in apoptosis of WT cells was investigated. The SK-NEP-1 cells were transfected with miR-21 inhibitor and cultured for $48 \mathrm{~h}$, followed by detection of apoptosis via flow cytometry. The results revealed that the ratio of apoptotic cells was markedly higher in miR-21 inhibitor group than that in NC group $(\mathrm{P}<0.05)$ (Fig. 2). 
A

C

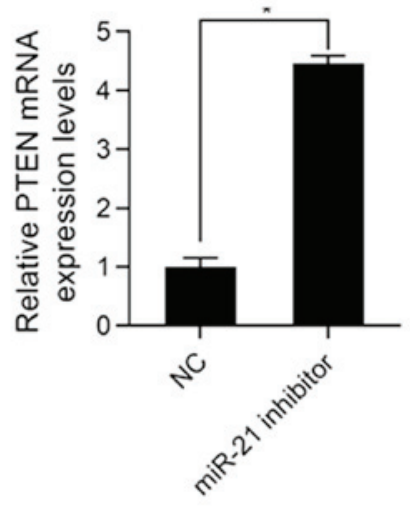

PTEN-3 'UTR-WT

miR-21

PTEN-3 ' UTR-MUT
B
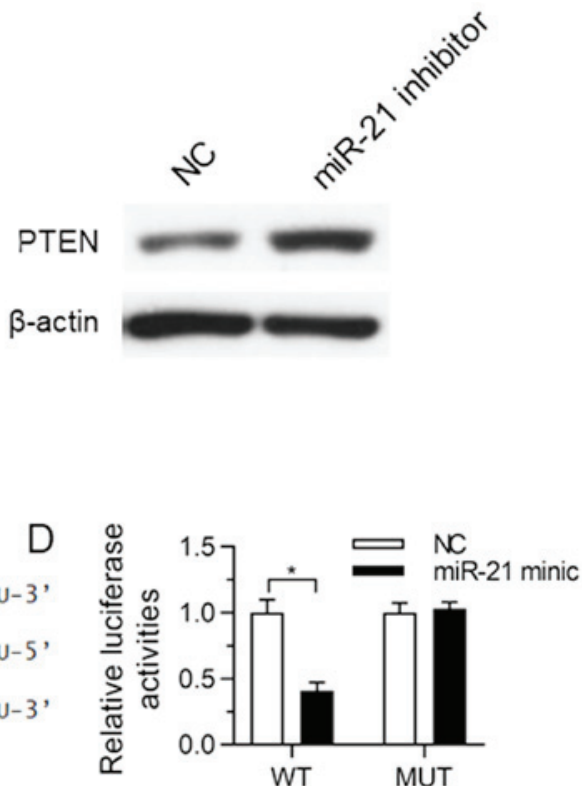

Figure 3. miR-21 binds to 3'-UTR of PTEN. (A and B) Effects of miR-21 repression on mRNA and protein expression levels of PTEN detected through RT-qPCR assay and western blot analysis. (C) Binding of miR-21 to 3'-UTR of PTEN predicted using software. (D) Luciferase activity in the cells co-transfected with PTEN 3'-UTR WT/MUT and miR-21 minic detected via dual luciferase reporter gene assay. ${ }^{*} \mathrm{P}<0.05$. miR, micro ribonucleic acid; UTR, untranslated region; PTEN, phosphate and tension homology deleted on chromosome ten; mRNA, messenger RNA; RT-qPCR, real-time quantitative polymerase chain reaction; WT, Wilms' tumor.

A

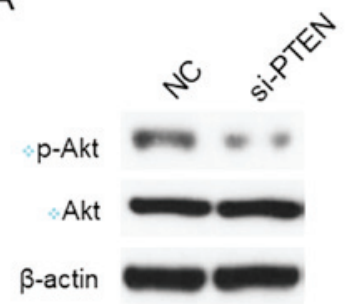

C

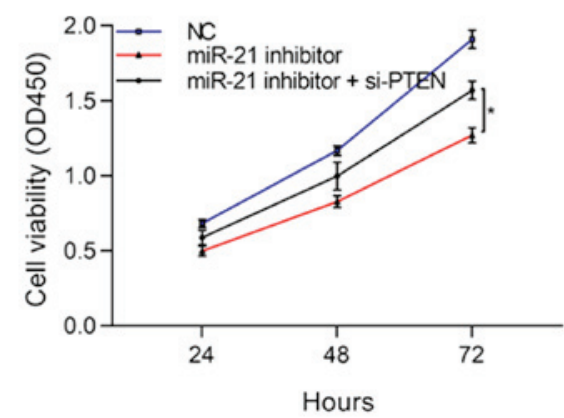

B

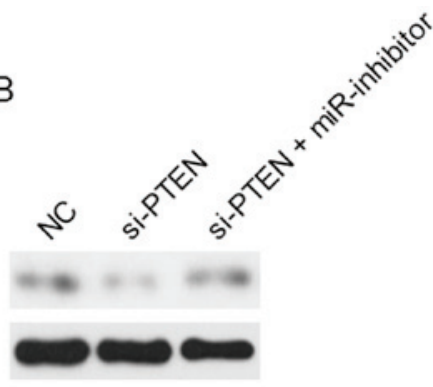

Figure 4. Correlation of miR-21 with PTEN and PI3K/Akt pathway. (A) Effects of silencing PTEN on p-Akt and Akt protein levels determined through western blotting. (B) Effects of concurrent silencing of PTEN and miR-21 on p-Akt and Akt protein levels detected by western blotting. (C) Involvement of PTEN in the inhibitory effect of miR-21 on proliferation of SK-NEP-1 cells detected via CCK-8 assay. ${ }^{*} \mathrm{P}<0.05$. miR, micro ribonucleic acid; PTEN, phosphate and tension homology deleted on chromosome ten; PI3K, phosphatidylinositol 3-kinase; Akt, protein kinase B; p-Akt, phosphorylated Akt; WT, Wilms' tumor; CCK-8, cell counting kit-8.

miR-21 bound to 3'-UTR of PTEN. Online TargetScan software was applied to analyze miRNA target genes, and it was discovered that PTEN was the most likely potential target of miR-21. The effects of miR-21 inhibition on PTEN expression was verified in SK-NEP-1 cells. After the SK-NEP-1 cells were transfected with miR-21 inhibitor and cultured for $72 \mathrm{~h}$,
RT-qPCR assay and western blot analysis were performed to detect the changes in PTEN at the mRNA and protein levels. The results uncovered that repressing miR-21 expression upregulated the PTEN expression at both the mRNA level (Fig. 3A, P<0.05) and the protein level (Fig. 3B), implying that miR-21 negatively regulates PTEN. To confirm that PTEN is 
a functional target of miR-21, the activity detection via dual luciferase reporter gene assay was carried out using the cells co-transfected with PTEN 3'-UTR WT/MUT and miR-21 minic (Fig. 3C). The miR-21 minic group exhibited inhibited luciferase activity of WT plasmids but unchanged luciferase activity of MUT plasmids, indicating that miR-21 suppresses the expression of PTEN by binding to PTEN 3'-UTR.

Correlation of miR-21 with PTEN and Akt pathway. The suppression of PTEN expression may have a relation to the Akt pathway. Therefore, the associations of miR-21 with PTEN and Akt were studied. The results showed that after silencing PTEN, the p-Akt level rose in SK-NEP-1 cells, but the Akt protein level had no obvious changes, suggesting that PTEN negatively modulates p-Akt. After silencing both PTEN and miR-21, the lower p-Akt was reversed, which thus reversed the inhibitory effect of miR-21 on the proliferation of SK-NEP-1 cells $(\mathrm{P}<0.05)$, implying that miR-21 inhibits the proliferation of the WT SK-NEP-1 cells through the PTEN/Akt pathway (Fig. 4).

\section{Discussion}

WT is usually detected in children aged $<5$ years, with an average age of onset of 38 months (2). WT is the most common malignant solid tumor in children, but it is widely regarded as one of the most treatable tumors due to modern comprehensive treatments in which analyzing sensitive molecular biomarkers to guide treatment and follow-up is playing a crucial role $(4,12)$.

Currently, extensive research has manifested that miRNAs can act as powerful cancer biomarkers (e.g., cancer type, prognosis and response to treatment) to play an important role in cell differentiation, proliferation, cell cycle control and apoptosis. More than $50 \%$ of human miRNA genes are located in vulnerable sites and regions and often associated with the development of cancers (13), which demonstrates the potential importance of miRNAs in cancers. In addition, miRNAs have been verified to serve as oncogenes or tumor suppressors and key molecules in the development and progression of cancers (14). As one of the overexpressed miRNAs most commonly found in solid tumors (10), miR-21 may act as an oncogene in the development and progression of cancers.

Increasing number of studies have proved that $(15,16)$ miR-21 is an attractive target in the multi-regulation of tumor genetics and pharmacology. Overexpression of miR-21 in pancreatic endocrine and acinar tumors is closely correlated with high Ki67 (a cell proliferation index) and liver metastasis (17). Analysis of the effects of anticancer chemotherapeutic agents such as 5-fluorouracil and gemcitabine on miRNAs has demonstrated that repressing miR-21 increases the sensitivity to gemcitabine-induced apoptosis, whereas miR-21 expression is increased after responding to the process with 5-fluorouracil (18). Besides, miR-21 is also detected in biological fluids such as serum. In comparison with healthy control samples, serum samples from patients with diffuse large B-cell lymphoma exhibit highly expressed miR-21. Moreover, the miR-21 expression in disease samples has an association with recurrence-free survival, but it is not related to overall survival. These methods highlight the potential application of miRNAs in cancers as non-invasive diagnostic markers (19). In this study, it was discovered that silencing miR-21 inhibited the proliferation of WT SK-NEP-1 cells and induced apoptosis.

PTEN was originally discovered to participate in the development of various diseases as a suppressor gene. PTEN knockdown is able to accelerate cell proliferation. Researchers have found that PTEN inhibits Akt activity by repressing PI3K activity. Akt participates in angiogenesis and metastasis and partially prolongs survival signals (20). The lack of PTEN may result in the sustained activation of the signaling pathway, thus losing the control of cell growth. Given this, PTEN upregulation is capable of promoting cardiomyocyte apoptosis, while its inactivation can reduce apoptosis (21). Previous studies have reported that apoptosis can eliminate harmful substances in cells, accordingly respond to cell invasion, provide energy for subcellular structure production and metabolism, and even maintain cell stability. The activated PI3K/Akt signaling pathway has important effects on the differentiation, proliferation and apoptosis of smooth muscle cells and vascular fibroblasts (22). A recent study (23) showed that miR-21 may target the key proteins of the PTEN/PI3K/Akt signaling pathway to mediate the proliferation, apoptosis, migration and invasion of human esophageal cancer cells and cell cycle progression. In this study, it was confirmed through the dual luciferase reporter gene assay that miR-21 bound to PTEN 3'-UTR to inhibit PTEN expression. Next, the associations of miR-21 with PTEN and PI3K/Akt signaling pathway were investigated, and it was uncovered that silencing PTEN elevated the p-Akt level, but had no great impact on the Akt protein level, suggesting that PTEN negatively modulates p-Akt. After silencing both PTEN and miR-21, the decrease in $\mathrm{p}$-Akt was reversed.

In conclusion, the results of this study suggest that miR-21 mediates the proliferation repression in the WT SK-NEP-1 cells through the PTEN/Akt pathway.

\section{Acknowledgements}

Not applicable.

\section{Funding}

No funding was received.

\section{Availability of data and materials}

All data generated or analyzed during this study are included in this published article.

\section{Authors' contributions}

$\mathrm{XZ}$ and LG designed the study and performed the experiments, XZ and CL collected the data, LG and HL analyzed the data, XZ and LG prepared the manuscript. All authors read and approved the final manuscript.

\section{Ethics approval and consent to participate}

Not applicable. 


\section{Patient consent for publication}

Not applicable.

\section{Competing interests}

The authors declare they have no competing interests.

\section{References}

1. Liu G, Zhang Y, Fu K, Hu J, Zhao Z, Fu W and Liu G: Meta-analysis of the effect of preoperative chemotherapy on Wilms' tumor. J BUON 23: 211-217, 2018.

2. Davidoff AM: Wilms' tumor. Curr Opin Pediatr 21: 357-364, 2009.

3. Liu CL, Wang WH, Sun YL, Zhuang HW, Xu M, Chen HF and Liu JX: miR-144-3p inhibits the proliferation and metastasis of pediatric Wilms' tumor cells by regulating Girdin. Eur Rev Med Pharmacol Sci 22: 7671-7678, 2018

4. Grundy PE, Breslow NE, Li S, Perlman E, Beckwith JB, Ritchey ML, Shamberger RC, Haase GM, D'Angio GJ, Donaldson M, et al; National Wilms Tumor Study Group: Loss of heterozygosity for chromosomes $1 \mathrm{p}$ and $16 \mathrm{q}$ is an adverse prognostic factor in favorable-histology Wilms tumor: A report from the National Wilms Tumor Study Group. J Clin Oncol 23: 7312-7321, 2005.

5. Rivera MN and Haber DA: Wilms' tumour: Connecting tumorigenesis and organ development in the kidney. Nat Rev Cancer 5: 699-712, 2005.

6. Gammell P: MicroRNAs: recently discovered key regulators of proliferation and apoptosis in animal cells: Identification of miRNAs regulating growth and survival. Cytotechnology 53: 55-63, 2007.

7. Ludwig N, Werner TV, Backes C, Trampert P, Gessler M, Keller A, Lenhof HP, Graf N and Meese E: Combining miRNA and mRNA expression profiles in Wilms tumor subtypes. Int J Mol Sci 17: 475, 2016

8. Jiang X and Li H: miR-1180-5p regulates apoptosis of Wilms' tumor by targeting p73. OncoTargets Ther 11: 823-831, 2018.

9. Liu GL, Yang HJ, Liu B and Liu T: Effects of microRNA-19b on the proliferation, apoptosis, and migration of Wilms' tumor cells via the PTEN/PI3K/AKT signaling pathway. J Cell Biochem 118: 3424-3434, 2017.

10. Krichevsky AM and Gabriely G: miR-21: A small multi-faceted RNA. J Cell Mol Med 13: 39-53, 2009.

11. Asangani IA, Rasheed SA, Nikolova DA, Leupold JH, Colburn NH, Post S and Allgayer H: MicroRNA-21 (miR-21) post-transcriptionally downregulates tumor suppressor Pdcd4 and stimulates invasion, intravasation and metastasis in colorectal cancer. Oncogene 27: 2128-2136, 2008.
12. Gratias EJ, Jennings LJ, Anderson JR, Dome JS, Grundy P and Perlman EJ: Gain of 1q is associated with inferior event-free and overall survival in patients with favorable histology Wilms tumor: A report from the Children's Oncology Group. Cancer 119: 3887-3894, 2013.

13. Selcuklu SD, Donoghue MT and Spillane C: miR-21 as a key regulator of oncogenic processes. Biochem Soc Trans 37: 918-925, 2009.

14. Deng S, Calin GA, Croce CM, Coukos G and Zhang L: Mechanisms of microRNA deregulation in human cancer. Cell Cycle 7: 2643-2646, 2008.

15. Ren Y, Zhou X, Mei M, Yuan XB, Han L, Wang GX, Jia ZF, $\mathrm{Xu}$ P, Pu PY and Kang CS: MicroRNA-21 inhibitor sensitizes human glioblastoma cells U251 (PTEN-mutant) and LN229 (PTEN-wild type) to taxol. BMC Cancer 10: 27, 2010.

16. Meng F, Henson R, Wehbe-Janek H, Ghoshal K, Jacob ST and Patel T: MicroRNA-21 regulates expression of the PTEN tumor suppressor gene in human hepatocellular cancer. Gastroenterology 133: 647-658, 2007.

17. Roldo C, Missiaglia E, Hagan JP, Falconi M, Capelli P, Bersani S, Calin GA, Volinia S, Liu CG, Scarpa A, et al: MicroRNA expression abnormalities in pancreatic endocrine and acinar tumors are associated with distinctive pathologic features and clinical behavior. J Clin Oncol 24: 4677-4684, 2006.

18. Rossi L, Bonmassar E and Faraoni I: Modification of miR gene expression pattern in human colon cancer cells following exposure to 5-fluorouracil in vitro. Pharmacol Res 56: 248-253, 2007.

19. Lawrie CH: MicroRNAs and haematology: Small molecules, big function. Br J Haematol 137: 503-512, 2007.

20. Panigrahi AR, Pinder SE, Chan SY, Paish EC, Robertson JF and Ellis IO: The role of PTEN and its signalling pathways, including AKT, in breast cancer; an assessment of relationships with other prognostic factors and with outcome. J Pathol 204: 93-100, 2004

21. Mocanu MM and Yellon DM: PTEN, the Achilles' heel of myocardial ischaemia/reperfusion injury? Br J Pharmacol 150: 833-838, 2007.

22. Gregorian C, Nakashima J, Le Belle J, Ohab J, Kim R, Liu A, Smith KB, Groszer M, Garcia AD, Sofroniew MV, et al: Pten deletion in adult neural stem/progenitor cells enhances constitutive neurogenesis. J Neurosci 29: 1874-1886, 2009.

23. Wu YR, Qi HJ, Deng DF, Luo YY and Yang SL: MicroRNA-21 promotes cell proliferation, migration, and resistance to apoptosis through PTEN/PI3K/AKT signaling pathway in esophageal cancer. Tumour Biol 37: 12061-12070, 2016. International (CC BY-NC-ND 4.0) License. 